\section{Modelling pre-trauma resilience and vulnerability factors for PTSD}

\section{R Parslow}

Orygen Research Centre, The University of Melbourne, Melbourne, Australia

Background: Research identifying vulnerability and resilience factors that may affect an individual's likelihood of developing post-traumatic stress disorder (PTSD) is hampered by the dearth of information on those who are resilient and, although exposed to the trauma, remain unaffected by it.

Methods: Such a study eventuated in Canberra where the Centre for Mental Health Research is conducting a longitudinal study of over 7000 participants from three age groups. Reinterviewed participants were asked about their level of exposure and reaction to this trauma and their fire-related PTSD symptoms. Information on a range of sociodemographic, health and personality measures was collected both before and after the trauma.

Results: Almost $80 \%$ of Wave 2 respondents were exposed to the fire, while around 2000 reported having experienced fire-related PTSD symptoms in the week prior to their interview. Structural equation modeling of pre-trauma risk and resilience factors associated with PTSD symptoms was undertaken. When level of exposure and immediate reaction to the fire were taken into account, pre-trauma resilience measures had the greatest impact on PTSD symptoms. Those with higher levels of resilience were significantly less likely to report PTSD symptoms.

Conclusion: These findings indicate that reducing risk of PTSD by increasing individuals' levels of resilience in the face of specific traumas has the potential to be an effective strategy to limit the negative psychological impact of trauma exposure.

\section{Clozapine invokes the EGF system to activate ERK: a novel target in treatment resistant schizophrenia?}

\section{A Pereira, G Fink, S Sundram}

Mental Health Research Institute of Victoria, Melbourne, Australia

Background: The atypical antipsychotic drug clozapine is superior to all other drugs in treatment of refractory schizophrenia. This is likely to involve clozapineinducing long-term neuronal adaptations contingent on drug-receptor activation of intracellular signaling to affect gene transcription. One candidate intracellular signaling pathway is the mitogen-activated protein kinase-extracellular signal-regulated kinase (ERK) cascade. This pathway regulates synaptic proliferation and plasticity, processes impaired in schizophrenia. We have previously reported that although clozapine and haloperidol acutely inhibited ERK activation in cortical neurons, only clozapine stimulated ERK with continued treatment. However, this stimulation was not through the canonical dopamine D2-Gi/o-PKA or the serotonin 5HT2A-Gq-phospholipase C-linked signaling pathways. Thus, we examined alternative signaling pathways that clozapine could mobilize to activate ERK including growth factor receptor systems.

Methods: Clozapine-induced phosphorylation of ERK1/2 in the absence or presence of growth factor receptor-specific inhibitors was measured in primary murine cortical cultures by Western immunoblotting. Results were normalized against vehicle and total ERK1 and 2 levels.

Results: The epidermal growth factor (EGF) receptor inhibitor, AG1478, caused significant dose-dependent inhibition of pERK1 (IC50 $0.083 \mu \mathrm{M}$ ) and pERK2 (IC50 $0.106 \mu \mathrm{M})$ in the presence of clozapine, whereas the platelet-derived growth factor receptor inhibitor, tyrphostin, A9 did not.

Conclusions: This is the first evidence that the effects of clozapine may involve a neuronal signaling system previously not linked to antipsychotic drug action. This presents a novel series of targets for exploration in the development of new therapeutics and insights into the pathology of schizophrenia.

\section{The prevalence of depression in the North West Adelaide Health Study}

\section{N Potts', D Wilson², A Taylor ${ }^{3}$, T Gill³, G Schrader4, R Ruffin ${ }^{2}$}

${ }^{1}$ University of Adelaide: ${ }^{2}$ Health Observatory, Department of Medicine, The Queen Elizabeth Hospital; ${ }^{3}$ Population Research and Outcome Studies Unit, South Australian Department of Health; and ${ }^{4}$ Department of Psychiatry, University of Adelaide, Adelaide, Australia

Background: The North West Adelaide Health Study is a population-based biomedical cohort study investigating the prevalence of chronic conditions and healthrelated risk factors. This study is based on a randomly selected group of individuals over the age of 18, who agreed to be involved in a longitudinal health study.

Methods: Over the past 18 months, 3488 participants were assessed. General demographic and socioeconomic data were collected. Participants were assessed for the presence of a number of chronic conditions including major depression. The presence of depression was assessed using the Center for Epidemiological Studies Depression Scale-D. A score of 16 or higher indicated mild depression. A score of 27 or higher was rated as moderate to severe depression. 
Results: Of the 3488 participants, $12.5 \%$ (436/3488) met criteria for depression. Of the population, $8.9 \%$ had a mild depression and 3.9\% met criteria for a moderate to severe depression. Depression was statistically significantly higher among women, adults living alone, those separated or divorced, and those unemployed, working part, casually employed or a student. Individuals with a degree or higher level of education, and those earning above $\$ 40 \quad 000$ were significantly less likely to have depression.

Conclusions: The demographics for those with depression are consistent with previously published studies of depression in the community. However, the rates of depression were higher than that seen in other South Australian, Australian and international studies. The possible reasons for this will be discussed.

\section{Use of the web in the self-management of bipolar disorder}

\section{J Proudfoot, G Parker, S Grdovic, L Greenfield, M Hyett}

The Black Dog Institute, Sydney, Australia

Background: Poor treatment adherence in people with bipolar disorder is common, and $40 \%$ relapse within a year. Education and support assist sufferers to selfmanage their condition and reduce hospitalizations, but the challenge lies in providing them in a convenient, private and nonstigmatizing manner.

Computer-based programs are acceptable to patients and efficacious, but debate exists about whether additional support is needed to maximize effects. This paper reports a study evaluating a web-based education program for people with bipolar disorder.

Methods: Developed at the Black Dog Institute by health professionals, community representatives and consumers, the Bipolar Education Program aims to help individuals and their families better understand the condition, recognize early warning signs, reduce triggers, develop self-management skills and enhance treatment compliance. Modules involve audiovisual presentations, questions and answers, advice from wellknown people with bipolar disorder and printouts.

A randomized controlled trial (RCT) is being conducted, comparing three versions of the program (on its own, with email support from an informed person, with informed support plus assistance to implement a 'staywell' plan) to minimal online education. Measures are being taken at pre-, post-, 3 and 6 months follow-up of clinical and psychosocial outcomes, and costs.

Results: Since its May 2006 launch, the Web site has recorded approximately 2000 visits per month. About $75 \%$ of first-time visitors have been women, 55\% aged 31-50, 44\% with bipolar disorder (predominantly $\leq 12$ months since diagnosis), $28 \%$ carers/family/friends and $18 \%$ health professionals. The RCT is about to commence, data from which will be presented at the conference.

\section{Keeping Care Complete: results from the first international schizophrenia and bipolar disorder family caregivers survey}

\section{R Ramjan \\ Schizophrenia Fellowship of NSW Inc., Sydney, Australia}

Background: The international shift toward community care and the deinstitutionalization of people with mental illness has seen much of the responsibility for day-to-day care transferred to family members. This responsibility can be associated with profound psychosocial, physical and financial impact. Keeping Care Complete is the first large, international survey of carers of people with schizophrenia or bipolar disorder.

Method: About 100 Australian carers were enrolled in an eight-country survey of 982 carers of people with schizophrenia or bipolar disorder. Participants were asked a series of questions regarding treatment, treatment discontinuation, relapse, satisfaction with treatment and carer burden. This was done by means of a 30-min telephone interview.

Results: Australian carers are much involved in their family member's treatment: $80 \%$ of those surveyed reported being the primary carer, $42 \%$ spent more than $20 \mathrm{~h}$ /week caring for their relative and 19\% were responsible for administering medications. For 97\% of carers, family support was the key factor, as well as medication, for keeping their family member well and $70 \%$ of the participants were frustrated by the low expectations for long-term improvement set by health care professionals.

Conclusion: This survey provides significant insights into the experiences of carers of people with mental illness and helps quantify the impact of this responsibility on carers in terms of their physical, mental and financial well-being.

\section{A randomized controlled trial to assess the efficacy of omega-3 fatty acids as a treatment for depression during the perinatal period}

\section{A-M Rees, G Parker, M-P Austin}

The Black Dog Institute, Sydney, Australia 